\title{
Visual Quality Measures for Characterizing Planar Robot Grasps
}

\author{
Eris Chinellato, Member, IEEE, Antonio Morales, Member, IEEE, Robert B. Fisher and \\ Angel P. del Pobil, Member, IEEE
}

\begin{abstract}
This paper presents and analyses twelve quality measures that characterize robotic grips according to their stability and reliability. The measures are designed to assess three-finger grips of $2 \mathrm{D}$ parts performed in a real environment, taking into account both theoretical aspects and unavoidable uncertainties of a grasping action. They build on the existing literature and on physical and mechanical considerations. The measures constitute a feature space that pattern recognition methods can use in order to classify robotic grips according to their quality. Six of the measures depend on the actual finger configuration of the gripper, and they have shown to be critical for a better characterization. The kinematics of the Barrett Hand has been used. As a validation step, the measures are merged in two global quality values (with different practical applicability) that can be used to rank feasible candidate grips.
\end{abstract}

Index Terms-Intelligent robots, Real time systems, Feature extraction, Stability criteria, Reliability estimation.

\section{INTRODUCTION}

O NE OF the main challenges in the research field of dextrous manipulation is to decide how to grasp unknown objects. This can be done by firstly producing a representation of the object to grasp, obtained by visual or tactile feedback. The representation can then be used to decide how to grasp the object. Many different candidate grips can be generated, and it is thus critical to characterize the quality of grips in order to execute the most reliable ones. We conceived and realized a set of twelve quality measures that assess the reliability of a three-finger planar grip according to different aspects, building on the rich existing literature, on physical and mechanical considerations and on the information given by practical experiments with a humanoid robot. The measures constitute a feature space suitable to be used by pattern recognition methods in order to classify robotic grips according to their quality, as we have done in [1], [2]. In this paper the word feature also stands for quality measure.

Our quality measures are designed to be used in a real environment, where uncertainties and noise need to be taken into account, in contrast with approaches which favors theoretically optimal grasps without considering their practical realization. Six of the features are directly concerned with the kinematics of the robot hand - the Barrett Hand in our case - whilst the hand attributes are usually ignored in the literature. In our grasp characterization we also integrate grips in which two

E.Chinellato, A.Morales and A.P.del Pobil are with the Robotic Intelligence Laboratory, Computer Science Department, Jaume I University, Castellón, Spain (e-mail:eris,morales,pobil@icc.uji.es)

R.B.Fisher is with the School of Informatics, University of Edinburgh, Edinburgh, UK (e-mail:rbf@inf.ed.ac.uk) fingers act as one virtual-finger; these are normally analyzed separately from three-finger grips.

We present here a practical validation in which the features have been used in a merged way to rank feasible grips according to their quality. More detailed results, both theoretical and experimental, are presented in a companion paper [1]. The validation step, followed by a stability analysis, has allowed us to draw some additional considerations about the features and their validity, but also constitutes an actual, applicable instrument to select between real robotic grips.

\section{Previous Research}

The problem of generating or choosing grasps of two dimensional objects is a classical one in robotics [3]. In our case, we use objects that are solid extrusions of 2D shapes. Also, we focus on force-closure grasps [4].

The heuristic quality measure introduced by Park and Starr [5] for both two and three-finger grips has been an important source of inspiration. Nevertheless, that paper focused more on defining grips for geometric shapes using both edges and vertices as contact points. In our case the objects are real, imperfect, not exactly modelled, and grips involving the vertices of the shapes are not allowed.

Mirtich and Canny [6] assume friction contacts and rounded fingertips and define the optimum planar grip as the one that best resists forces and torques about the grip plane. The best three-finger grasp of a 2D object is the equilateral grasp having the largest outer triangle, and the best two-finger grasp has the two forces opposing along the maximum chord of the object.

Xiong et al. [7] propose a quantitative measure for evaluating what they call the dynamic stability of a grasp, obtained using the Lyapunov stability concept and assuming soft fingertips and rolling contacts. Though their approach is rather analytical, some of the concepts they introduce have been useful in defining the final set of features.

Looking for stable grasps of polygonal shapes, Ponce and Faverjon [8] use two measures: the distance of a contact point from the margin of its grasping region and the distance of the center of the grasp from the centroid of the shape. Both are used in the present work with the necessary adaptations.

The restrictions of [9] are too specific to be directly applied to our needs, but this paper has been useful as a source of inspiration, such as, in minor extent, [10] and [11].

A complementary approach, based on affine augmentation, was developed in the nineties [12].

Finally, much research is focused on two-finger grips only (e.g. [13], [14], [15]), as they usually require a different kind 
of analysis. Such research has also been considered in order to successfully merge two and three-finger grip characterization.

The work presented here builds on research carried on at the Jaume I University. In [16] the background of the whole grasping system is introduced, and the grasping problem specifically analyzed with particular attention to the kinematics and geometry of the Barrett Hand. We did not develop analytical validation of our measures or comparisons with previous metrics, as our interest focuses more on empirical approaches and practical applications.

\section{BACKGROUND}

\section{A. Grasps}

We describe a grasp with the set of three contact points between hand and object contour, and the corresponding force directions, perpendicular to the contour, which meet in the grasp force focus. We call hand configuration each possible grip obtained applying the kinematics constraints of a hand to a grasp. Hereinafter, when referring to grasps and configurations together, the term grip is used.

We assume a real-time system acting in an unstructured environment, which detects unknown objects and, through analysis of visual data, selects and executes a stable grip of such objects.

Fast computation is necessary in order to achieve a real-time interaction with the external world. The ability to cope with uncertainties, in terms of knowledge of friction coefficients or visual and positioning errors, is a must in an uncontrolled environment.

The main stages of a robotic grasping system of this kind are the following:

1) produce an image of an unknown planar object, extract its contour and identify triplets of grasping regions;

2) determine a number of feasible, force-closure grasps selecting the grasping points for each region triplet;

3) generate finger configurations that could actually be applied to the object in order to perform a grip action;

4) characterize the candidate hand configurations with a number of significant features;

5) using those features, perform an 'intelligent' selection of a grip to execute;

6) execute the grip with support of visual and tactile feedback.

Our research, which is more widely described in [17], investigates a new approach for step 4, and consequently step 5. Details about the other sections of a system of this kind, concerned with the generation of candidate grasping configurations, are given in [16].

\section{B. Configurations}

With a perfectly homogeneous hand, for which the fingers are all the same, the three possible ways of combining fingers with contact points in a grasp are not distinguishable. This is not the case for the Barrett Hand, for which the kinematics of the thumb is different from that of the other two fingers. A photo of the hand is reproduced in Fig. 1(a). Its kinematics are

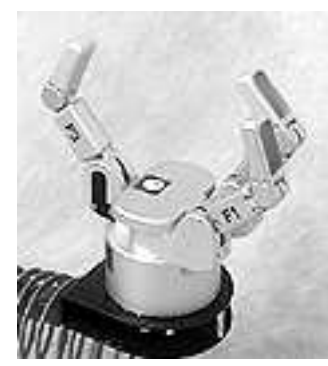

(a) Photo

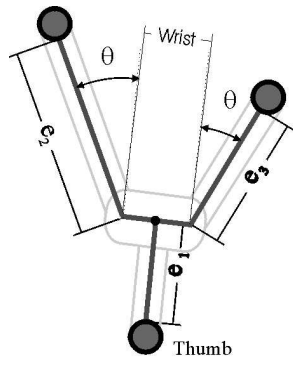

(b) Kinematics
Fig. 1. Barrett Hand, http://www.barretttechnology.com

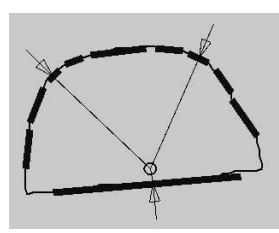

(a) Grasp

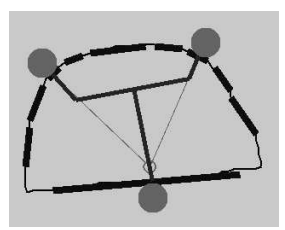

(b) Configuration 1

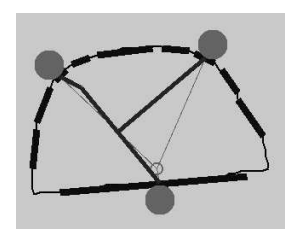

(c) Configuration 2
Fig. 2. Generating configurations from a grasp

depicted in Fig. 1(b). The hand has four degrees of freedom: the three finger extensions $e_{1}, e_{2}, e_{3}$ and the spread angle $\theta$.

For each grasp there are three possible positions of the thumb. After deciding where to place the thumb, there are still infinite ways of making the hand touch the object at three contact points. However, when the action line of the thumb is fixed as well, only one solution is possible. A one-dimensional search along all possible thumb force directions gives the best Barrett Hand configuration for a grasp after the thumb position has been defined (the force-line criterion is used to carry out this choice process). Thus, every grasp ideally generates three different configurations, one for each thumb position. When no solutions are found for a thumb position within a grasp, due to the constraints deriving from the hand geometry and kinematics, no corresponding configurations are produced.

Typically, dozens of configurations can be generated for an object, mostly depending on the number of regions found. In Fig. 2(b) and 2(c) two configurations generated from the grasp of Fig. 2(a) are depicted.

\section{Two-finger grips}

A particular kind of three-finger grasp is obtained as an extension of two-finger grasps. To generate a two-finger grasp, only two regions are needed, and they must be nearly parallel and facing each other (with friction, regions that are not perfectly parallel can also be used for two-finger grips).

Starting from a real two-finger grasp, if one of the regions is large enough to carry two Barrett Hand fingers, then a virtual two-finger grasp is generated. So, there is a special group of three-finger grasps that are computed in a completely different way, and thus have different properties and characteristics. 


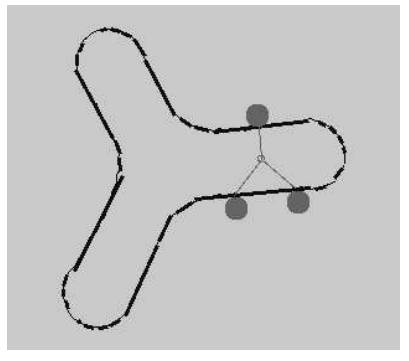

(a) Grasp

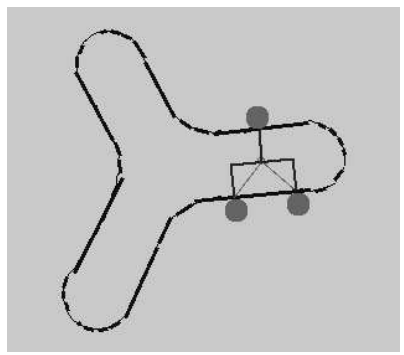

(b) Configuration
Fig. 3. Example of two-finger grip

From now on we will refer to them as two-finger grasps, meaning that two of the fingers are positioned on the same grasping region.

Each two-finger grasp can generate only one configuration, that is a two-finger configuration, as the thumb must be the finger opposed to the other two. An example of a two-finger grasp and its configuration are shown in Fig. 3 (a) and (b).

\section{GRIP DESCRIPTORS}

\section{A. Description of a grip}

All the descriptors of grasps and configurations that are used to implement the quality features are explained below. Some descriptors are common, others are peculiar to either grasps or configurations. For clarity, they are presented here in different groups and illustrated in Fig. 4, Fig. 6 and, for two-finger grip descriptors, Fig. 5.

SHARED DESCRIPTORS:

- grasping regions. The portions of the object contour where the three fingers are placed. They are modelled as short straight segments and described by the coordinates of their extreme points. In Fig. 4 they are the thicker zones on the object contour.

- region curvature. All points of a region are assumed to have approximately the same curvature value. The value used is the average of the single local curvatures.

- contact points. The three points where the fingers are supposed to touch the object, each lying on one of the three grasping regions $\left(P_{1}, P_{2}, P_{3}\right.$ in Fig. 4 and 5). They define the grasping triangle, whose sides are the segments joining the grasping points. For simplicity, we assume point contacts with friction, although features $\mathrm{S} 4, \mathrm{C} 3$ and C6 take into account the fact that real contacts are not points but small surfaces.

- triangle center. It is the centroid of the grasping triangle (the triangle formed by the contact points). It is used only for two-finger grips assessment. In Fig. 5 is called $C_{T}$.

- area. The area of the object to grasp.

- centroid. It is computed as the geometrical centroid of the two dimensional shape described by the extracted object contour. It corresponds to the center of mass of the three dimensional, homogeneous object obtained by a normal extrusion of such a shape. Point $\mathrm{C}$ in Fig. 4.

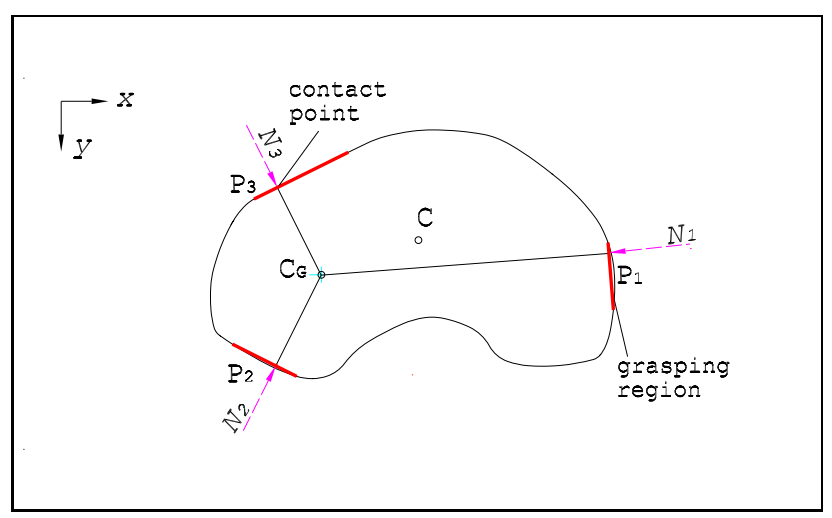

Fig. 4. Object and grasp descriptors

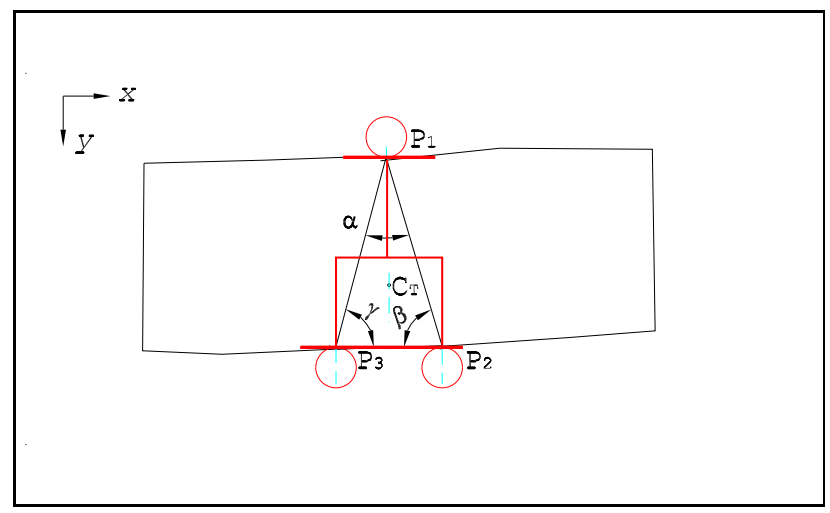

Fig. 5. Two-finger grip descriptors

- inertia axes. The major inertia axis is useful for a simplified physical representation of the object. The centroid of the object is the intersection of the major inertia axis with its minor.

\section{GRASP DESCRIPTORS:}

- force directions. The directions of the three vectors that correspond to the forces exerted by each finger on the object. In Fig. 4 they are represented with the arrows $N_{1}, N_{2}, N_{3}$. They are usually normal to the contour.

- force focus. To achieve force closure for the grasp, the vectors of the three forces need to meet in a point inside the intersection of the vector cones. This point is called force focus, and also referred to as the center of the grasp $C_{G}$ (see Fig. 4).

\section{CONFIGURATION DESCRIPTORS:}

- force directions. Same as for grasps, but this time the forces are the ones actually exerted by the fingers of the Barrett Hand, usually different from the ideal forces of the generating grasp. $F_{1}, F_{2}, F_{3}$ in Fig. 6.

- real force focus. The real center of a configuration $\left(C_{C}\right.$ in Fig. 6). It is the intersection of the directions of the real forces.

- finger extensions. The projected distances ( $e_{i}$ in Fig. 6) between the fingertips (more exactly the contact points) and the center of the hand. This is a measure of how open a finger is.

- finger spread. The opening angle of the two fingers in opposition to the thumb. They must have the same 


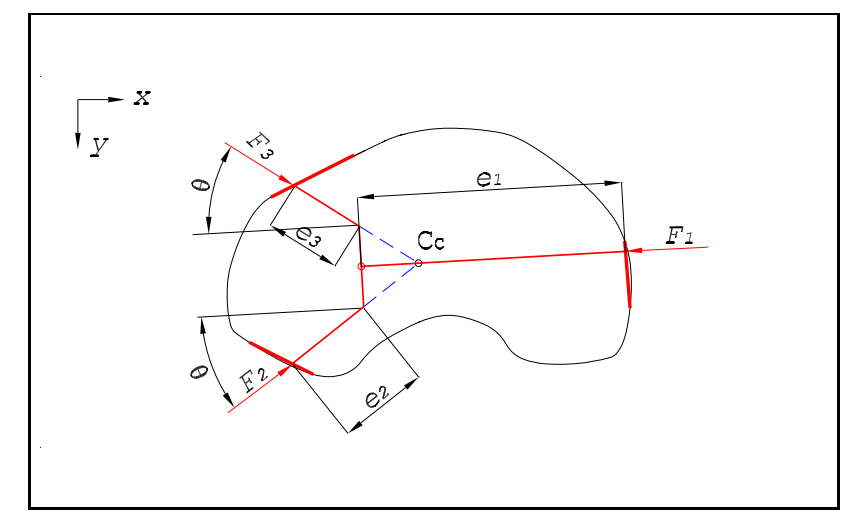

Fig. 6. Configuration descriptors

spreading ( $\theta$ in Fig. 6), limited between $0^{\circ}$ (perfect opposition) and $180^{\circ}$ (perfect alignment).

\section{FEATURES}

\section{A. Classification}

All the features we defined to characterize the grip quality are here described. As explained above, the descriptors used to implement the quality measures can be either common or peculiar to grasps or configurations. Hence, it is natural to divide the features in the same three categories:

$\mathrm{S}$ features - the ones that can be used to characterize both grasps and configurations;

$\mathrm{G}$ features - the ones only suitable for characterizing grasps;

$\mathrm{C}$ features - the ones for characterizing configurations.

The features of the first kind are related to the object, the contact points and the regions, as these are the descriptors in common between grasps and configurations (the $S$ in their codes stands for Shared).

\section{B. Grasping parameters}

Three global parameters have been introduced in our work, in order to give the system additional reliability and flexibility.

The first parameter is the friction coefficient $\mu$ of the contact between the fingers and the object. Since we will not know the exact value for this physical quantity, it is very reasonable to set a lower limit for it (to set friction 0 would equally be a non realistic assumption). For fingers covered with rubber, a static friction coefficient not lower than 0.4 can be reliably assumed regardless of the object material and its lubrication. In different conditions the minimum coefficient can be different, for example reduced down to 0.15 for greasy metal-metal contacts. The friction parameter must not vary during an experiment, but can be changed when needed according to macroscopic changes in the basic system conditions (e.g. a change of the finger material).

The second parameter is the finger positioning error threshold $\lambda$. When placing a finger on an object, the actual positioning correctness depends on the quality of the visual model of the object, and thus on brightness conditions, object colour, shadows and so on. We set an error threshold according to theoretical and practical considerations in order to distinguish between safe and risky finger placements. For safety reasons, the actual error threshold should be larger than the expected placement error. Hence, if the maximum error is likely to be around $1 \mathrm{~mm}$, the threshold could be set to $2 \mathrm{~mm}$, as has been done in this case.

The third parameter we define is the object weight index $W$. Throughout this research all objects are assumed to have an average weight, but in the awareness that some features, like the ones related to the mass center approaching, assume larger or smaller importance according to the weight of the object. This is the reason for the introduction of this third parameter, that can be changed when changing the object material.

These parameters can be the base for extensions of our approach, letting the system learn what the most appropriate values are, according to different environmental circumstances that can be detected by the sensory apparatus. The parameters used are summarized below. Their influence on the different features will be introduced within the features description.

$\mu$ - estimate of the minimum possible friction between fingers and object; we use 0.4 , corresponding to forces having a maximum deviation angle from the normal of about $22^{\circ}$.

$\lambda$ - estimate of the maximum possible finger positioning error; using $2 \mathrm{~mm}$, but higher values may be necessary in the practical applications.

W - estimate of object weight class (example of possible weight values: light $=0.5$, medium $=1$, heavy $=2$ ); the medium weight 1 is always used here. A different, more appropriate estimate could be chosen by learning or feedback.

\section{Normalization}

We wanted to have all features comparable and defined over similar ranges. For a grip, all features are defined so as to have have theoretical best value of 0 (except for S2 and S4, that are lower bounded to strictly positive values) and middle quality 1 . This has been obtained by normalizing some features, according to physical aspects, and relying on a 'halfway' method: a grip which is halfway between the best and the worst possible assessment will have a normalized quality of 1 . The normalization also allows to compare three and two finger grips, for which some features need to be defined in different ways.

\section{Shared features}

S1. POINT ARRANGEMENT: According to [5] and [6], a three-finger grip is more reliable in terms of stability, sliding avoidance and force equilibrium when it is closer to an ideal equilateral grip. Each three finger grip can thus be characterized with a value intended to measure the similarity of its grasping triangle to an equilateral one.

We adopt the most obvious implementation of this feature, proposed by Park and Starr [5], in which each angle is compared with a $60^{\circ}(\pi / 3 \mathrm{rad})$ angle typical of an equilateral triangle (Fig. 7):

$$
Q_{S 1}=\frac{3}{2 \pi}\left(\left|\alpha-\frac{\pi}{3}\right|+\left|\beta-\frac{\pi}{3}\right|+\left|\gamma-\frac{\pi}{3}\right|\right)
$$




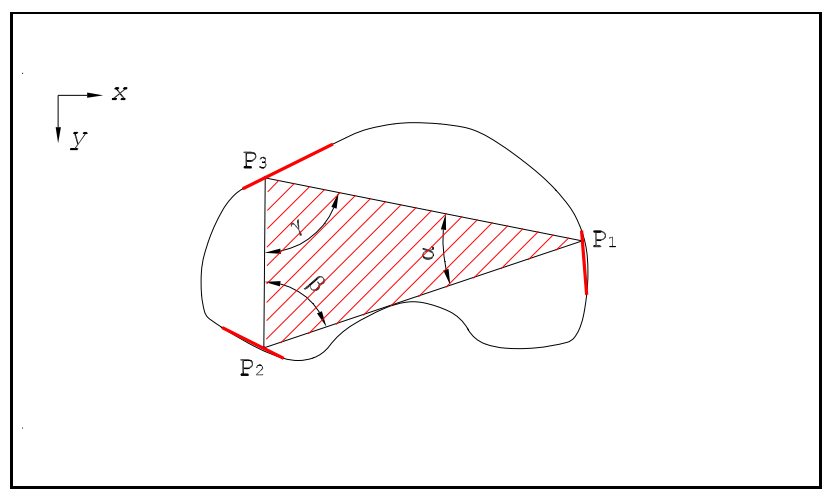

Fig. 7. Features S1 - point arrangement, S2 - triangle size

With the coefficient $3 / 2 \pi$, the minimum possible value is 0 for a perfectly equilateral grasping triangle, 2 for the worst case of a triangle degenerating to a segment and 1 for a halfway condition.

The implementation of this feature for two-finger grips is the following, $\alpha$ being the angle at the thumb contact point, and $\beta, \gamma$ being the angles at the base of the triangle (Fig. 5):

$$
Q_{S 1}^{\prime \prime}=\frac{2}{\pi}(|\beta-\gamma|+\alpha)
$$

The first term assesses the deviation of the grasping triangle from an ideal isosceles triangle, obtained when $\beta=\gamma$. The second term is aimed to assess the optimality of the grip. As the length of the triangle base is fixed when the two opposition fingers join each other in a two-finger grip, the angle $\alpha$ is an index of the distance between the thumb and the other two fingers: the smaller the angle, the longer the distance, and the better the grip [6]. Again, the coefficient $2 / \pi$ makes the variation range of the feature to be ]0,2], according to what happens to three-finger grips.

S2. TRIANGLE SIZE: As a conclusion of their dynamic stability analysis, Xiong et al. [7] state that, the larger the area of the grasping triangle, the more stable a grip is. This condition is also similar to what was proposed in [6]. Intuitively, given a force, the torque produced is higher the longer the force distance. Thus, the larger the grip, the greater is the torque that is possible to resist using the same force. The area of the grasping triangle is represented in Fig. 7.

The quality value must then be inverse to the area $A_{T}$ of the triangle formed by the three contact points:

$$
Q_{S 2}=W \frac{A}{4 A_{T}}
$$

The reason for such implementation is the following. Considering an equilateral triangle, the ideal way of grasping it is putting the three fingers on the middle of its sides, thereby obtaining a grasping triangle that has area $1 / 4$ of the original triangle area. So, calling A the area of the object, an average grasping triangle is assumed to have an area equal to $\mathrm{A} / 4$. This actually gives average normalized quality values close to 1 .

Physical considerations suggest that the grasping triangle size is more important for heavy objects, where stability against gravitational and inertial torques is a critical issue. Hence, to consider the effect of the weight of the objects, the coefficient W (object weight class) is included in the formula.

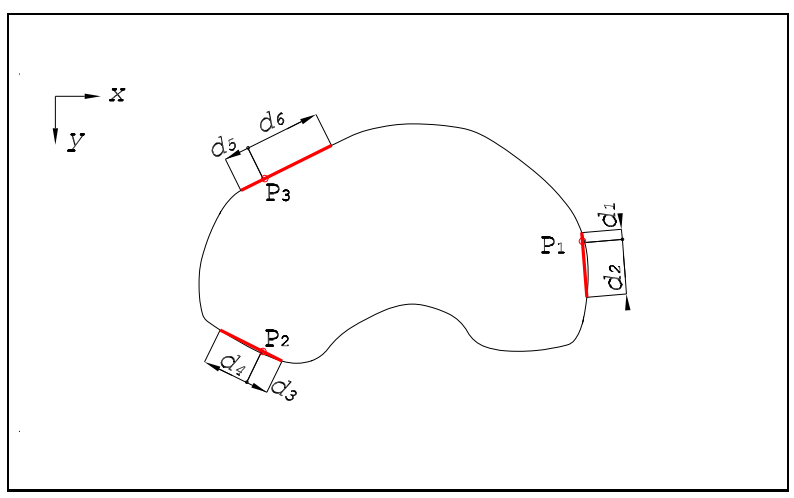

Fig. 8. Feature S3 - grasping margin

S3. GRASPING MARGIN: Finger positioning is not free from uncertainties, due to both vision imperfections and mechanical tolerances. Therefore, when the contact points are close to the extremes of a grasping region, the fingers are more likely to fall outside of the region itself. This can completely modify the quality of a grip, as the assumptions on the force directions can decay.

Hence, the safety threshold $\lambda$ has been defined to use in this feature. For a contact point farther than the threshold from the region margin, there are nearly no chances of placing a finger outside of the region, and the penalty is 0 . For contacts nearer to the margin than the threshold, the lower the distance from the margin, the higher the risk of getting out of the region.

The feature is implemented by considering all the six distances $d_{i}$ of each contact point from the extremes of its grasping region (see Fig. 8), and summing all the corresponding misplacement risks:

$$
Q_{S 3}=\sum_{i=1}^{6} q_{i} \quad q_{i}= \begin{cases}0 & \text { for } d_{i} \geq \lambda \\ \frac{\lambda}{d_{i}}-1 & \text { for } d_{i}<\lambda\end{cases}
$$

The threshold $\lambda$ needs to be larger than the expected positioning error. A sensible way to set is to estimate the positioning error and multiply it by 1.5 or 2 . The value used here for the threshold is $\lambda=2 \mathrm{~mm}$, but a best value can only be assigned to $\lambda$ after experimental validation.

S4. CONTACT CURVATURE: Humans normally prefer to grasp objects on concave contours than on convex ones. It is analytically demonstrated [10] that when the contacts are made on concave surfaces (being sure that there is enough space to place the finger) the grips are more stable. Accordingly, this feature takes into account the local curvature of the grasping regions at the contact points.

An index of a grasping region curvature is obtained by averaging the data on the local curvature of each point on the region. The curvature values are positive for concave surfaces, negative for convex surfaces, and ideally 0 for perfectly flat surfaces. In Fig. 9 the top left region will have a curvature index very close to 0 , and the other two regions will have slightly negative curvature values. We define the overall grip quality as 1 minus the sum of the three region curvature values:

$$
Q_{S 4}=1-\left(\rho_{1}+\rho_{2}+\rho_{3}\right)
$$




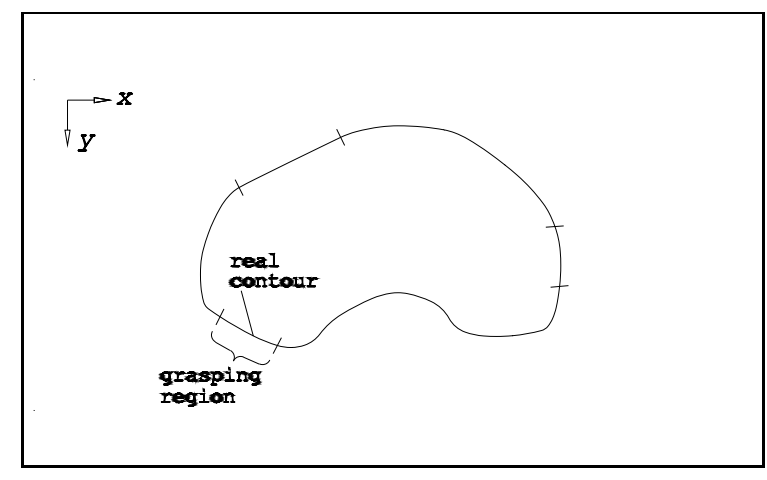

Fig. 9. Feature S4 - contact curvature

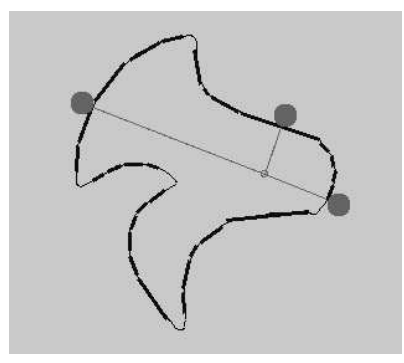

Fig. 10. Example of critical situation for feature G1

The average quality value of 1 is given to grips having all three grasping regions ideally flat. Such implementation always provides results between 0 and 2 (the allowed curvature is bounded during the grip generation process).

\section{E. features for grasp assessment}

G1. FORCE ARRANGEMENT: The best vector-closure condition for the equilibrium of a grasp (see [5], [9]) is attained when the force directions are uniformly distributed around the object ( $\phi_{1}=\phi_{2}=\phi_{3}$ in Fig. 11). In other words, the three angles between the forces should all be as close as possible to $120^{\circ}$. This penalizes wide and narrow angles, favoring grasps with more balanced forces, that are also more reliable against uncertainties in the actual direction of the forces applied.

Park and Starr called this feature 'arrangement of force directions'. The implementation they proposed is the following:

$$
Q=\left|\phi_{1}-2 \frac{\pi}{3}\right|+\left|\phi_{2}-2 \frac{\pi}{3}\right|+\left|\phi_{3}-2 \frac{\pi}{3}\right|
$$

However, this implementation is not always good, as it doesn't consider the risk given by forces lying nearly in the same direction (see Fig. 10, where two forces nearly opposing each other constitute a big threat to the stability of the grip).

In situations like this, there is a serious risk of losing the force-closure condition, as the force vectors are close to a position in which they wouldn't span the plane anymore. Our implementation respects the optimality of the most uniform grasps (when $\phi_{1}=\phi_{2}=\phi_{3}=120^{\circ}$ the best assessment of 0 is obtained), strongly penalizing, at the same time, grasps with forces that are separated by angles close to $180^{\circ}$ :

$$
Q_{G 1}=\frac{\pi^{3} / 27}{\left(\pi-\phi_{1}\right)\left(\pi-\phi_{2}\right)\left(\pi-\phi_{3}\right)}-1
$$

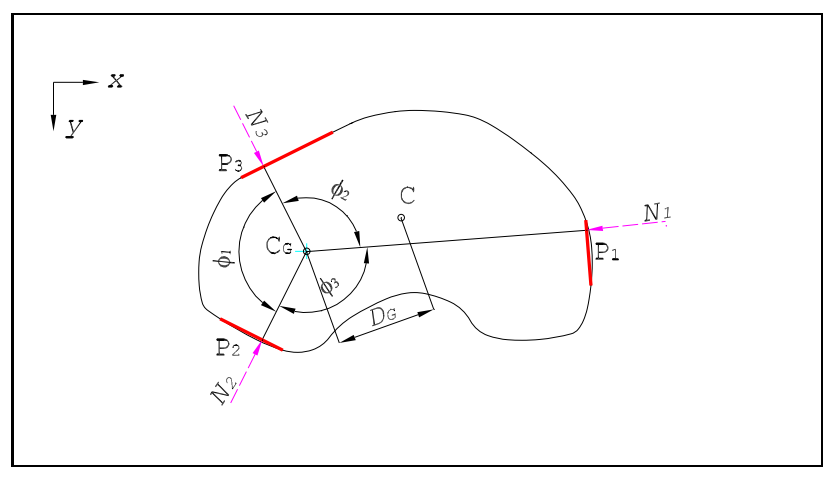

Fig. 11. Features G1 - force arrangement, G2 - focus centring

All two-finger grasps are given the optimal value of 0 according to this feature, as they do not present this kind of risk.

G2. FOCUS CENTRING: This feature is designed to obtain stable grips with respect to wrenches generated by gravitational and inertial forces. These wrenches are minimum when the center of the grip is closest to the mass center of the object. Dealing with objects that are three dimensional extrusions of two dimensional shapes, the mass center can be reliably projected on the centroid of the two-dimensional contour. Thus, the feature value takes into account the distance $D_{G}$ between the force focus of the grasp (assumed as its center) and the shape centroid $C$, as shown in Fig. 11.

Such distance assumes a greater influence when the object is heavier, as in this case it is more important to grasp the object close to the mass center, to reduce torques due to gravitational forces. For this reason, the object weight index parameter $W$ is used in the final formula of this feature, together with the average of the major and minor inertia semi-axes $M_{L}$ and $M_{L}$ (values that represent the object size):

$$
Q_{G 2}=2 W \frac{\left\|C_{G}-C\right\|}{M_{L}+m_{L}}
$$

A different analysis needs to be done for two-finger grasps, for which the three forces are not going to meet in a focus at all. The problem here is to decide what the center of the grip is. According to physical considerations, the point used as center of a two-finger grip is the center of the grasping triangle (see VIII-B for details on the reasons of this choice). Hence, to obtain the expression of feature G2 for two-finger grasps, we just substitute $C_{G}$ with the center of the grasping triangle $C_{T}$ (see Fig. 5):

$$
Q_{G 2}^{\prime}=2 W \frac{\left\|C_{T}-C\right\|}{M_{L}+m_{L}}
$$

\section{F. features for configuration assessment}

C1. FORCE LINE: Due to the particular geometry of the Barrett Hand, the actual direction of the forces exerted by the three fingers in a given configuration is not usually the ideal one corresponding to the original grasp. In Fig. 12 the deviations between theoretical forces $N_{i}$ and real forces $F_{i}$ are called $\delta_{i}$. As the friction coefficient of the contacts between object and fingers is not known beforehand, the more the 


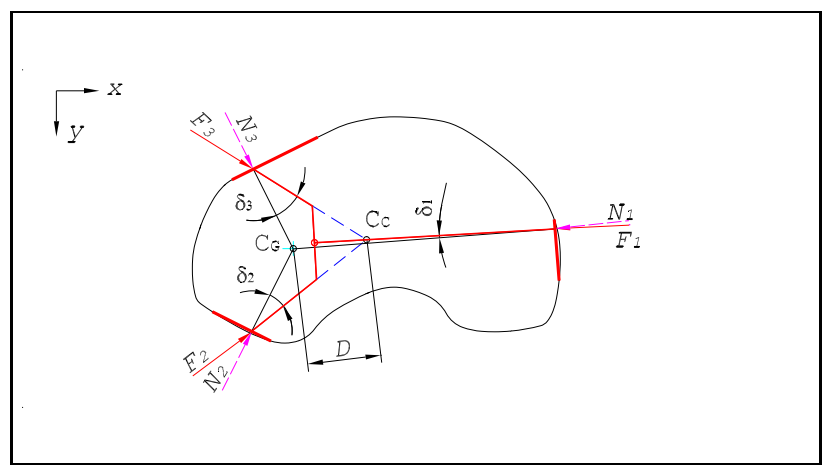

Fig. 12. Features $\mathrm{C} 1$ - force line, $\mathrm{C} 2$ - real focus deviation

forces deviate from the normal, the more the fingers risk sliding along the side of the object, due to a too high tangential component of the applied force.

This feature assesses such risk by summing the square values of the deviations of the actual force directions from the normal to the contour:

$$
\begin{gathered}
Q_{C 1}=\frac{4 k}{3}\left(\delta_{1}^{2}+\delta_{2}^{2}+\delta_{3}^{2}\right) / \arctan ^{2} \mu \\
k= \begin{cases}1 & \text { if } \forall i \quad \delta_{i} \leq \arctan \mu \\
3 & \text { if } \exists i \mid \delta_{i}>\arctan \mu\end{cases}
\end{gathered}
$$

The square values, rather than the absolute values, gives a stronger handicap to the largest of the three deviations, introducing a kind of intrinsic threshold in the feature. Though, a real threshold has been defined for this feature, as a configuration with even one finger pointing out of the friction cone can never be considered reliable, even if it is very good according to other aspects.

The threshold is set according to presumptions about the friction of the contact between fingers and object. So, having chosen a threshold friction coefficient $\mu$ (typically between 0.1 and 0.4), the corresponding threshold angle is $\nu=\arctan \mu$. The constant $k$ in the formula is normally equal to 1 , and it is set to 3 when a configuration has one or more of the finger forces with a deviation from the normal higher than the threshold angle.

The feature is normalized so that the value of 1 is given to a configuration having all its deviation angles equal to half the threshold angle.

C2. REAL FOCUS DEVIATION: The real focus deviation feature accompanies and completes the force line feature. The first is more concerned about the deviation of the single forces from the normal, that could end in forces being out of the friction cone, thus compromising the stability of a contact. This feature is aimed more at assessing the total deviation of a configuration from the generating grasp. In fact, the original force directions are computed in order to optimize the vector closure, and it is thus useful to try and assess how much a configuration is sub-optimal according to this aspect.

We assess this deviation with the distance $D$ between the configuration real focus from the theoretical one (Fig. 12):

$$
Q_{C 2}=\frac{\left\|C_{G}-C_{C}\right\|}{\eta \mu / 2}
$$

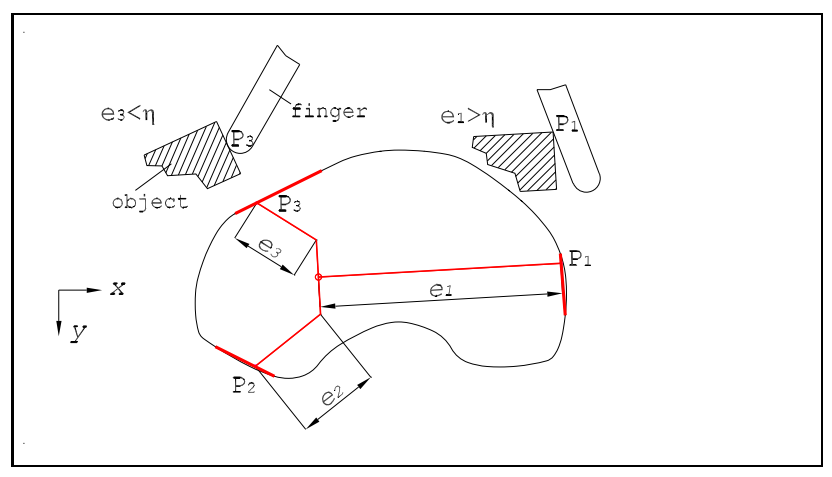

Fig. 13. Features C3 - finger extension, C6 - finger limit

The real focus $C_{C}$ is the intersection point of the real force directions (the symmetry of the finger spread assures its existence). The farther it is from the theoretical focus $C_{G}$, the higher is the risk that it lies outside the closure zone of the grasp, given by the intersection of the friction cones. This would strongly affect the overall stability of the grip.

The theoretical maximum deviation is the maximum finger extension $\eta$ multiplied by the friction threshold $\mu$, thus the normalized formula makes the feature value range from 0 to 2 , with the usual middle quality of 1 . As explained above, the center of both two-finger grasps and two-finger configurations is the center of the grasping triangle, so that the deviation for two-finger grips is always 0 .

C3. FINGER EXTENSION: A finger having only two joints can contact the side of the object in different ways according to its extension. If two fingers act on an object with two different extensions, they would touch the object in two positions having slightly different distances from the surface, and thus they would probably exert a torque out of the horizontal plane of the object. Clearly, this is not desirable and the purpose of this feature is to define how much a configuration can suffer from this kind of problem.

The task is to compare the differences in the finger extensions, as fingers with the same extensions are supposed to act more uniformly on the object, thus minimizing the risk of unwanted torques. This can be done summing the square differences between the three extensions (see Fig. 13):

$$
Q_{C 3}=\frac{1}{\eta^{2}}\left(\left(e_{1}-e_{2}\right)^{2}+\left(e_{2}-e_{3}\right)^{2}+\left(e_{3}-e_{1}\right)^{2}\right)
$$

The coefficient $1 / \eta^{2}$ is half of the possible maximum value for the above sum, so that again the feature is defined in $[0,2]$.

This feature is useful for another reason: different finger extensions can generate contact zones having different size and shape. Such unevenness can provoke different friction conditions to the three contacts with consequent instability.

C4. FINGER SPREAD: According to [5], a good, equilibrated grip should have its three forces roughly equally separated by three $120^{\circ}$ angles. A sort of force arrangement feature has already been implemented in the present work for assessing grasps (G1), and the theoretical discussion made there is still valid. Though, the application to configuration assessment requires some more considerations. 


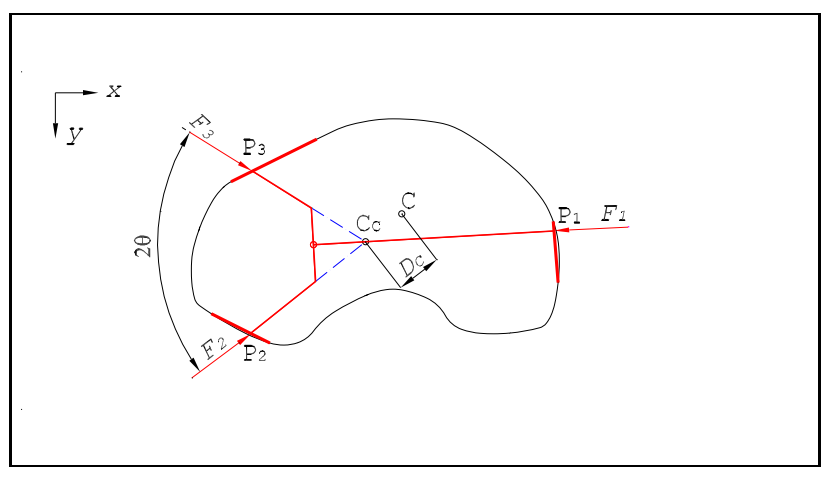

Fig. 14. Features C4 - finger spread, C5 - real focus centring

The finger spread feature $\mathrm{C} 4$ tries to adapt the arrangement of force directions to the special geometry of the Barrett Hand. In its first implementation [16], the value was given by the deviation of the opening angle of the fingers from the ideal value of $60^{\circ}(\pi / 3)$ (which gives three forces equally separated by $120^{\circ}$ angles). In this way, all configurations having the thumb nearly opposed to the other two fingers are given a bad quality, same as for configurations having the other two fingers nearly in opposition. This last situation is the only one that should be carefully avoided and thus strongly penalized, being close to a condition of non-closure, as explained for feature G1 and shown in Fig. 10. Configurations with small opening angles, as in the case of two-finger grips, are instead not bad at all for the particular geometry of the Barrett Hand.

According to such considerations, the following is thus the chosen implementation of this feature, depicted in Fig. 14:

$$
Q_{C 4}= \begin{cases}0 & \text { for } \theta \leq \frac{\pi}{3} \\ \frac{\frac{\pi}{6}}{\frac{\pi}{2}-\theta}-1 & \text { for } \theta>\frac{\pi}{3}\end{cases}
$$

The best value of 0 is obtained by all configurations with a spread smaller or equal to $\pi / 3$ (thus including the two-finger grips), whilst for spreads approaching $\pi / 2$ the rate is going to infinity.

C5. REAL FOCUS CENTRING: This feature is the correspondent of the mass center approaching feature G2, this time applied to configurations. Again its purpose is to minimize the effect of gravitational and inertial forces. The real focus, i.e. the intersection of the actual force directions, is used as center of the configuration.

The formula is the same as in G2, but now the used distance is $D_{C}$ of Fig. 14:

$$
Q_{C 5}=2 W \frac{\left\|C_{C}-C\right\|}{M_{L}+m_{L}}
$$

The center of the configuration for a two-finger grip is the center of the grasping triangle, and the feature changes accordingly:

$$
Q_{C 5}^{\prime \prime}=2 W \frac{\left\|C_{T}-C\right\|}{M_{L}+m_{L}}
$$

For the other elements in the formulas, the explanation given in G2 still applies.
C6. FINGER LIMIT: This feature has been introduced to take into account a particular geometrical aspect of the Barrett Hand. When the hand closes in on objects, there is a finger extension value that, if exceeded, causes the grip to be more risky and less stable, although still possible. Such a situation is shown in the upper right corner of Fig. 13 and compared with a proper secure grip on the upper left of the image.

This means that, even if a configuration is theoretically executable, it may not actually be reliable. Therefore, a threshold on the optimal maximum finger extension has been set in order to avoid fingerside contacts. Configurations having one or more fingers above this extension value are strongly penalized, as good contacts are not guaranteed.

The implementation of feature C6 thus is:

$$
Q_{C 6}=\epsilon_{1}+\epsilon_{2}+\epsilon_{3} \quad \epsilon_{i}= \begin{cases}e_{i}-\eta & \text { for } e_{i}>\eta \\ 0 & \text { for } e_{i} \leq \eta\end{cases}
$$

where $e_{i}$ are the finger extensions and $\eta$ is the optimal maximum finger extension, which has been analytically computed for the Barrett Hand, giving a value of $101.5 \mathrm{~mm}$. Nevertheless, practical experience suggests that the actual value can be slightly different from this.

\section{TwO GLOBAL QUALITY INDEXES}

The natural way to verify the validity of the defined features is to use them for predicting the reliability of real robotic grips. Nevertheless, we wanted to find a way of first validating the features theoretically without the need of a robot. Especially, we are interested in understanding if the features based on the hand do provide additional useful information in order to characterize a grip. For this purpose, we define two global quality values: $Q_{G}$ includes all $G$ features suitable to assess grasps, $Q_{C}$ all $C$ features designed to assess the configurations. The shared features $S$ are used for both indexes.

Several different pattern recognition methods can be used to merge the features in a single value. Nevertheless, we preferred a method that gives all features the same influence on the final result, that is, we decided to simply sum the value of all features and obtain a numerical index that is the quality of a grip. This was possible thanks to the decision of giving the features similar ranges and setting a theoretical best value of 0 and a middle quality value of 1 . Thus, these are the simple implementations of the two indexes:

$$
\begin{gathered}
Q_{G}=S_{1}+S_{2}+S_{3}+S_{4}+G_{1}+G_{2} \\
Q_{C}=S_{1}+S_{2}+S_{3}+S_{4}+C_{1}+C_{2}+C_{3}+C_{4}+C_{5}+C_{6}
\end{gathered}
$$

There are $4+2=6$ features for grasp assessment and $4+6=10$ features for configuration assessment.

The two overall quality indexes allows to rank all grasps and configurations available for an object.

\section{A. Definition and evaluation of the features}

During the whole process of defining and studying the quality features, different analyses have been performed on the single features and the final results, in order to better 
understand the nature of the problem and the significance of the solution proposed.

The whole configuration evaluation and selection process has been applied to nine different objects. Other trials have been done on extra shapes and different views of the objects.

Statistical instruments have been used to compare and assess the features, in order to define the final set and the merging step. From the beginning, the distribution of each implemented feature has been plotted and compared with the distributions of other features and that of the overall quality indexes. As a consequence of this analysis, some features have been changed in their implementation, to better achieve more uniformity in the whole feature set.

\section{B. Statistical correlation and other instruments}

Statistical correlation was used to check for patterns of agreement between features. Here is an example of correlation values between features and betwen each feature and the configuration global quality index, computed for one of the shapes (similar results were obtained for other shapes):

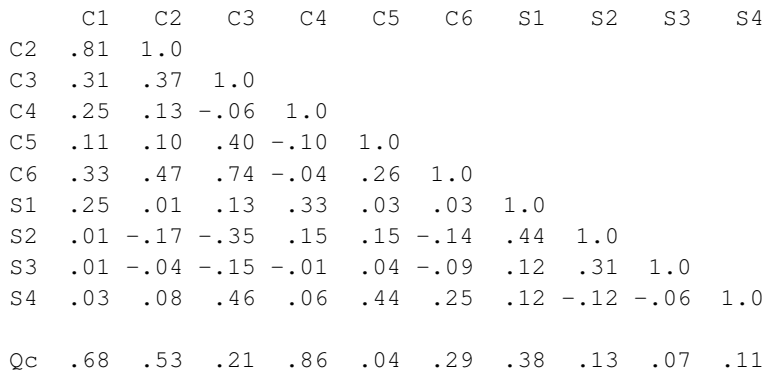

The values shown are typical, but correlations vary as different shapes emphasize different features [17]. The analysis suggests that: 1) none of the features are perfectly correlated and thus redundant, 2) many of the features are nearly uncorrelated, suggesting that they are assessing different aspects and 3) the global quality is partly correlated with the individual features, as one would expect, but also has some independent character, which means it should perform a better characterization than each feature individually.

The results section of this paper is much reduced for space reasons. More details, including results for other shapes, stability analysis, effects of parameter tuning and exhaustive experimental results, are included in a companion paper [1].

\section{RESULTS}

\section{A. Comparison between configuration and grasp characteri- zation}

The first way of analyzing the results is to compare the configuration rank with the grasp rank, trying to understand the relation between them. A routine has been implemented capable to match each grasp with all the configurations deriving from it.

In Tab. I, an example of the grasp quality computation is given (the shape is the same for which we showed the correlation indexes). The first 10 grasps in the rank are included, with their overall quality $Q_{G}$ in the second column, and the ranks of the configurations obtained from each of them

\begin{tabular}{|r|c|r|r|r|}
\hline $\begin{array}{r}\text { Grasp } \\
\text { Rank }\end{array}$ & $Q_{G}$ & $\begin{array}{r}\text { Config. 1 } \\
\text { Rank }\end{array}$ & $\begin{array}{r}\text { Config. 2 } \\
\text { Rank }\end{array}$ & $\begin{array}{r}\text { Config. 3 } \\
\text { Rank }\end{array}$ \\
\hline 1 & 2.061 & 12 & 14 & \\
2 & 2.130 & 2 & 20 & 5 \\
3 & 2.563 & 1 & 9 & 15 \\
4 & 2.741 & 3 & 22 & 31 \\
5 & 2.774 & 13 & 24 & 25 \\
6 & 2.929 & 18 & & \\
7 & 2.955 & 8 & 30 & 38 \\
8 & 2.972 & 10 & 21 & 47 \\
9 & 3.000 & 26 & 29 & 58 \\
10 & 3.213 & 16 & 46 & \\
\hline
\end{tabular}

TABLE I

COMPARISON BETWEEN GRASP AND CONFIGURATION ASSESSMENT

in the last three columns. As it can be seen, the configurations generated by the best grasp are only $12^{\text {th }}$ and $14^{\text {th }}$ in the rank, while the first configuration belongs to the third grasp.

A cross check between the ranks shows that the best configuration belongs to one of the best three grasps for 7 out of 8 shapes. On the other hand, once again for 7 cases out of 8 , the best configuration of the best grasp is between the first and $6^{\text {th }}$ position in the final configuration rank.

Therefore, a good theoretical triplet of contact points and force directions often gives rise to a good practical grip, but not in all the cases. The cases in which a very good grasp originates bad configurations show that the features related to the hand can indeed provide very useful practical information for a robotic system, and that a good theoretical grip could be not successful at all in the real world if application aspects are not considered.

Even though a good grasp does not always generate a very good configuration, there is evidence that, in order to find a good grasping configuration, it is necessary to start from a good theoretical grasp. This information could be useful in order to first screen a large number of candidates before performing a finer evaluation.

Two correlation coefficients were computed using the rank of the grasps and the one of the related configurations. The first coefficient measures the correlation of the grasp rank with the rank of the best configurations derived from each grasp. The second measures the correlation of the grasp rank with the average rank of all configurations derived from each grasp. Both values are meant to verify if a good grasp corresponds to a good configuration and vice versa. The correlation values found were usually similar, and varying between 0.6 and 0.8 depending on the object. This result, expressing a good but not perfect agreement between the two assessments, confirms that to find a good configuration we should look between good grasps, but also that the hand features are critical in order to properly characterize a real grip.

\section{DISCUSSION}

The results obtained allow us to make some additional observations about the set of features. Significance, applicability and possible improvements of each feature are here discussed, offering a better understanding of all of them, especially on where and how they should be used. Most of the features could 


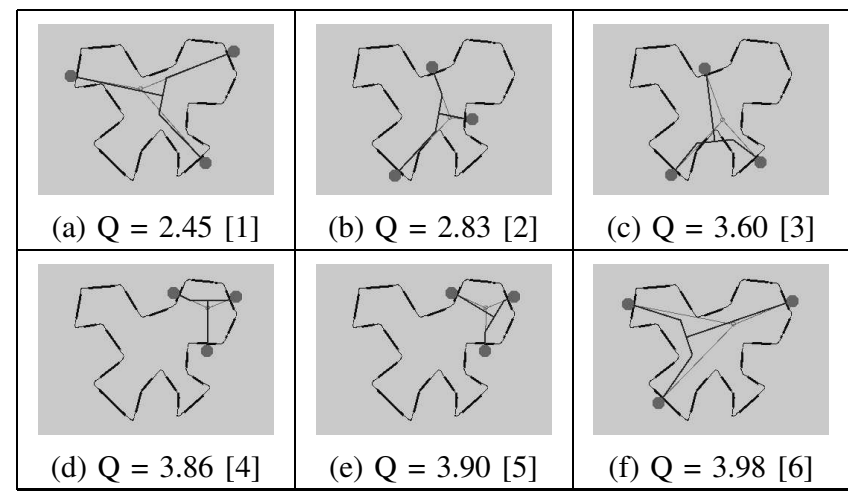

Fig. 15. Best grips with merged quality values. Synthetic shape.

be implemented in different ways, while still respecting the underlying theoretical inspiration. Some interesting variations that could bring remarkable changes in the characterization performance are proposed.

\section{A. Shared Features}

S1. POINT ARRANGEMENT: This feature is well recognized in the grasping literature, and it is applicable to all kinds of three finger grips.

More controversial is its two-finger implementation. For sure, both aspects considered (symmetry and length) are important in assessing a two-finger grip. Less clear is how they should be balanced, and how they should balance the three finger implementation. Anyway, even though the implementation of both aspects in this feature is arbitrary, the reasonable results obtained for the two-finger configuration assessment tell us that a good solution should take into account these aspects and give them the right importance in the assessment of a two-finger grip.

S2. TRIANGLE SIZE: This feature, similarly to G2 and its configuration version $\mathrm{C} 5$, evaluate the grip resistance to torques generated by gravitational and inertial forces, whose magnitude is determined in the first instance by the weight of the object, which appears in the formula. This is indeed valid for all kinds of grips and hands.

Considering together features $\mathrm{G} 2$ and $\mathrm{S} 2$, it is possible to say that the first aims to reduce the torque to be resisted, whilst the second increases the ability of the grip to resist torques. We expect that experiments with a robot will tell us that the area of the grip is definitely very important when the grip is not well centered, but not really critical when it is. An alternative feature merging S2 and G2 was used at an early stage of the implementation. The quality index according to this feature was given by the distance from the centroid divided by the area of the grasping triangle. In the final implementation it was decided to separate the two features once again, in order to give more importance to both, but the issue on how this aspect should be best implemented is still open.

S3. GRASPING MARGIN: The distance of a contact from the limit of a grasping region is a fundamental measure of the reliability of a grip. However, the way in which the grasping regions model the object contour strongly influences

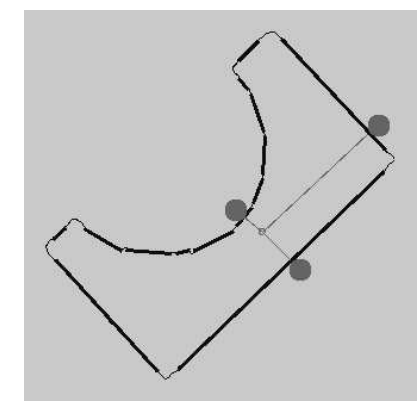

Fig. 16. Example of acceptable grasp penalized by feature G1

the type of characterization performed by the feature. In our implementation, a region can be segmented because of an excessive accumulated curvature from its first to its last point, or for a strong discontinuity in the curvature. In the first case there is no real risk in placing a finger near the margin or even outside the region, as it is going to touch the object in a place having very similar curvature value as the region has. On the other side, if there is a discontinuity, it is better to avoid it.

Even within the set of features implemented here, S3 is one of the most important. A method to distinguish the two kinds of margins hasn't been implemented so far, but it would be very important in order to better adapt the set of features to the real world.

S4. CONTACT CURVATURE: The importance of this feature is reinforced by its clear correspondence in human grasping choices. Nevertheless, it is very much influenced by how the information on the contour curvature is expressed. The ideal condition is to have reliable information on the curvature of the contact point and its close neighbors. The average region curvature used here is an acceptable solution but not as meaningful and reliable as more precise curvature estimates. An optional implementation, which would assure a best possible value of 0 , is to give penalty 0 both to flat and concave contact zones, and only penalize convex zones.

\section{B. Features for grasp assessment}

G1. FORCE ARRANGEMENT: Having forces symmetrically distributed around the object is an important condition for the equilibrium of a grip and this is well recognized in the literature. The issue is more about how much the grips should be penalized if they are far from the ideal condition. In this study, a solution that modifies what was originally done by Park and Starr [5] has been chosen. We give a high handicap to grips having two forces nearly facing each other, as there is in these cases a strong risk of losing the force-closure condition. Nevertheless, this is not always a big problem, for example when there are concave grasping regions that give extra stability to the grip (as in figure 16). Thus, to discern this kind of situation from the really risky one is very difficult, and requires a more complex analysis and implementation.

G2. FOCUS CENTRING: An interesting issue related to this feature is the one about its implementation for two-finger grasps. Since the forces are not meeting in a focus, deciding what the center of a two-finger grip is is not very obvious. Often in the literature the distance is computed from the 
centroid to the thumb force line. Nevertheless, this measure takes into account only one direction, and in this study something more precise was preferred, to use in conjunction with the distances computed for the three-finger grips.

The method we used to select the center was to specify a required force condition, the one in which the three forces are perfectly balanced, and see where the shape centroid should be to optimize such a condition. In the case of a two-finger grip, this happens when the centroid is at $2 / 3$ of the distance from the thumb to the middle of the grasping base. The physical proof for this is quite easy. To have an insight of this, it is enough to think that, if the centroid is half way between the thumb and the opposing fingers, the thumb force needs to be double than the other two forces, and this is certainly not an optimal condition. Thus, such a point is used as the center of a two-finger grip, and it is exactly the centroid of the grasping triangle, obtained by averaging the coordinates of the vertices.

A possible criticism to this solution is that the projection of the distance perpendicular to the thumb force line is more important that the tangential one. This observation could be fulfilled by giving more weight to one distance compared to the other.

A further issue related to this feature is that the distance from the centroid is probably a more critical issue for twofinger grips, rather than for three-finger grips. Two different weights could be given to the different situations, but all these improvements should be supported by practical proofs.

\section{Features for configuration assessment}

C1. FORCE LINE: Unlike the more general S and $\mathrm{G}$ features, the $\mathrm{C}$ ones are dedicated to the configuration assessment, and thus have an applicability range that is determined by the geometry of the hand.

Feature $\mathrm{C} 1$ is crucial for the grip characterization and is implemented here as a difference measure between real and ideal forces. Therefore, this feature is indeed of fundamental importance for all kinds of hands and grips.

A possible alternative implementation could disregard the coefficient $k$, in order to obtain a smoother quality distribution.

C2. REAL FOCUS DEVIATION: Despite its relation with C1, this feature gives a different measure of a grip stability.

The numerical results given by features $\mathrm{C} 1$ and $\mathrm{C} 2$ have been compared, to check their real autonomy. They are correlated, but they not totally dependent on each other, and for some grips they provide very different assessments. The reason is that the quality of a grip according to $\mathrm{C} 2$ is affected not only by the force deviations, but also by the finger extensions.

C3. FINGER EXTENSION: Feature C3 is indeed very important, as it copes with an aspect that cannot be disregarded when using the Barrett Hand for grasping. A grip which has a bad quality according to this feature is most probably not well balanced.

If the hand has fingers with three joints, this feature may be not as useful, as the third joint could provide the degrees of freedom necessary to give full flexibility for the exact contact position. Anyway, this can only be verified in a real environment depending on the particular hand used.
C4. FINGER SPREAD: This feature has been inspired by the concept of force arrangement. It is actually an adaptation of feature G1 to the particular kinematics of the Barrett Hand. The $60^{\circ}$ threshold used is arbitrary, but the handicap for finger spread slightly above the threshold is very low.

This feature is very important to check the reliability of a grip. Some configurations that were well assessed by other features despite being unsafe, have been strongly penalized by it. Nonetheless, it only applies to the Barrett Hand, or to hands with very similar geometry. For other hand geometries, feature G1, which has more general applicability and theoretical value, should be used instead of $\mathrm{C} 4$.

C5. REAL FOCUS CENTRING: Feature C5 is a different implementation of G2, applied to a hand geometry for which the real focus is different from the theoretical one. Its meaning is exactly the same, and all the considerations made for G2 are valid for $\mathrm{C} 5$ as well. Its applicability is clearly reduced to particular hand conformations.

C6. FINGER LIMIT: This feature implements the gradual worsening from a safe to an impossible grip due to increasing finger extension. Its applicability is very general, as the concept it fulfills is valid for all kinds of hands. The main issues are: (1) what is the finger extension limit that defines an impossible grip, (2) what is the limit that defines a perfectly reliable grip, and (3) how should the quality decrease between these two limits. Surely, these three points need to be defined according to the hand in use. A solution is here proposed for the Barrett Hand, and a possible improvement to it could be that of using the square of the differences to compute the final value, in order to avoid sudden changes. Anyway, only practical experiments can say the last word on how to manage the issue described above.

\section{CONCLUSION}

We presented a set of twelve features designed to characterize the quality of planar robotic grips. Such quality measures, though open to possible improvements in their implementation, do take into account all main aspects related to the reliability of a visual-based candidate grip.

The main causes of uncertainties influencing a grasping action are taken into account, and given strong importance within the features. The parameters introduced provide additional flexibility and customizability. Unlike most of the previous research, the hand geometry is given a great importance here, as six of the twelve features are specific for the Barrett Hand or for hands with similar kinematics. Efforts have been made throughout the project to successfully involve virtual twofinger grips in the analysis.

We presented a validation of the features within a method for selecting between many possible candidate grips. The system is capable of providing not only a rank to decide what grips to perform, but also a global quality index for each grip. Such a quality index can be used as a general measure to estimate and compare the reliability of grips, even for different objects and working environments. The hand features have proved to be critical in order to perform a better characterization of a real grip which takes into account the execution uncertainties and the working conditions. 
Further work has been developed which includes the use of the quality measures joined with experimental data gathered from real robotic grasping. The features have been used to predict the possible outcome of a grasping action [1], [2], and a comparison of the performance obtained with and without the hand features has been realized, confirming the theoretical and practical importance of such features.

Our future research plans include the improvement of the evaluation system with better 3D features (e.g. surface contacts), and the revision of some criteria paying attention to composite constraints.

\section{ACKNOWLEDGMENT}

This work was developed while the first author was in the University of Edinburgh, School of Informatics, and has been partially supported by the Student Awards Agency for Scotland (SAAS), by CICYT-DPI2001-3801, by Generalitat Valenciana (inf01-27, CTIDIA/2002/195, GV01-244), by Fundació CaixaCastelló (P1-1B2001-28), and by Spanish Ministerio de Ciencia y Tecnología (FPI program).

\section{REFERENCES}

[1] A. Morales, E. Chinellato, A. Fagg, and A. del Pobil, "Using experience for assessing grasp reliability," International Journal of Humanoid Robotics, in press.

[2] — , "Experimental prediction of the performance of grasp tasks from visual features," in IEEE Intl. Conf. on Intell. Robots and Syst., 2003, pp. 3423-3428.

[3] J. Boissonnat, "Stable matching between a hand structure and an object silhouette," IEEE Trans. on PAMI, vol. 4, no. 6, pp. 603-612, 1992.

[4] V.-D. Nguyen, "Constructing force-closure grasps," Intl. J. Robotics Research, vol. 7, no. 3, pp. 3-16, 1988.

[5] Y. Park and G. Starr, "Grasp synthesis of polygonal objects using a three-fingered robot hand," Intl. J. Robotics Research, vol. 11, no. 3, pp. 163-184, 1992.

[6] B. Mirtich and J. Canny, "Easily computable optimum grasps in 2-d and 3-d," in IEEE Intl. Conf. on Robot. Automat., 1994, pp. 739-747.

[7] C.-H. Xiong, Y.-F. Li, and H. Ding, "On the dynamic stability of grasping," Intl. J. Robotics Research, vol. 18, no. 9, pp. 951-958, 1999.

[8] J. Ponce and B. Faverjon, "On computing three-fingered force-closure grasps of polygonal objects," IEEE Trans. Robot. Automat., vol. 11, no. 6, pp. 868-881, 1995.

[9] X. Markenscoff and C. Papadimitriou, "Optimum grip of a polygon," Intl. J. Robotics Research, vol. 8, no. 2, pp. 17-29, 1989.

[10] D. Montana, "The condition for contact grasp stability," in IEEE Intl. Conf. on Robot. Automat., 1991, pp. 412-417.

[11] J. Trinkle, "On the stability and instantaneous velocity of grasped frictionless objects," IEEE Trans. Robot. Automat., vol. 8, no. 5, pp. 560-572, 1992.

[12] J. Dai and D. Kerr, "Analysis of force distribution in grasps using augmentation," J. Mech. Eng. Sci., Proc. IMechE, Part C, vol. 210, no. 1, pp. 15-22, 1996.

[13] J. Ponce and B. Faverjon, "On computing two-finger force-closure grasps of curved 2d objects," in IEEE Intl. Conf. on Robot. Automat., 1991, pp. $424-429$.

[14] I.-M. Chen and J. Burdick, "Finding antipodal point grasps on irregularly shaped objects," IEEE J. Robot. Automat., vol. 9, no. 4, pp. 507-512, 1993.

[15] D. Montana, "Contact stability for two-fingered grasps," IEEE Trans. Robot. Automat., vol. 8, no. 4, pp. 421-430, 1992.

[16] A. Morales, P. Sanz, A. del Pobil, and A. Fagg, "An experiment in constraining vision-based finger contact selection with gripper geometry," in IEEE Intl. Conf. on Intell. Robots and Syst., 2002, pp. 1711-1716.

[17] E. Chinellato, "Robust strategies for selecting vision-based planar grasps of unknown objects with a three-finger hand," MSc Thesis, Division of Informatics, University of Edinburgh, UK, 2002. [Online]. Available: http://www3.uji.es/ eris/MScThesis.pdf 